\title{
The Influence of Ion Implantation on the Optical Parameters - Refraction and Extinction Coefficients of the Oxygen-Enriched Layers Covering GaAs Implanted with Indium Ions
}

\author{
W. Rzodkiewicz ${ }^{a, *}$, M. KuliK $^{b}$, K. PyszniaK ${ }^{b}$ And A.P. KobzeV ${ }^{c}$ \\ ${ }^{a}$ Institute of Electron Technology, al. Lotników 32/46, 02-668 Warsaw, Poland \\ ${ }^{b}$ Institute of Physics, Maria Curie-Skłodowska University, pl. M. Curie-Skłodowskiej 1, 20-031 Lublin, Poland \\ ${ }^{c}$ Frank Laboratory of Neutron Physics, Joint Institute for Nuclear Research, 141980 Dubna, Russia \\ The semi-isolating GaAs (100) samples irradiated with fluence $3 \times 10^{15}$ ions $/ \mathrm{cm}^{2}$ of $\mathrm{In}^{+}$ions were character- \\ ized by using the methods: Rutherford backscattering spectroscopy, nuclear reaction analysis and ellipsometric \\ spectroscopy. The values of the thicknesses layers enriched with oxygen and the implanted were determined by the \\ methods of nuclear reaction analysis and Rutherford backscattering spectroscopy. Multilayer models were applied \\ for determination of the optical constants (refraction and extinctions coefficients) of investigated samples. The \\ thickness of native oxide covering the surface of implanted GaAs and refraction coefficients were increased after \\ implantation with indium. The spectrum of extinction indexes as a function of light wavelength has two bands \\ near the light wavelengths $400 \mathrm{~nm}$ and $480 \mathrm{~nm}$. The observed effects can be interpreted as formation of local \\ oxides of In and InAs precipitates or ternary alloys in enriched with oxygen layers at the surfaces of implanted GaAs.
}

PACS numbers: 61.72.uj, 78.66.Qn, 82.80.Yc

\section{Introduction}

The study of the damage produced in GaAs implanted with noble gases and indium have been recently reported [1-5] and also an increasing interest has been observed in the formation of InAs/GaAs quantum dots [6]. The process of diffusion of indium in implanted GaAs were presented [7]. In these investigations the methods, such as Rutherford backscattering spectroscopy (RBS), channeling ellipsometry with one-wave light and ellipsometric spectroscopy (ES) were used. There were observed creations of amorphous layer and changing optical constants refraction and extinction coefficients of implanted layer. In [8, 9] it was assumed that the optical parameters of oxide layer covering the implanted surfaces were the same as for the virgin samples. It was presented that the thickness and chemical composition of an oxide layer on GaAs surface irradiated with $\mathrm{Ar}^{+}$and $\mathrm{Xe}^{+}$depend on implantation fluencies [10]. In this paper we present the continuation of the study of GaAs implanted with indium. The optical parameters refraction and extinction coefficients of oxides which cover the implanted surfaces of GaAs are discussed in the investigations. The measurements were performed using the following methods: RBS, nuclear reaction analysis (NRA), and ES.

\section{Experiment}

The surfaces of semi-isolating GaAs (100) samples have been implanted with $200 \mathrm{keV} \mathrm{In}{ }^{+}$ions using the UNIMAS ion implanter of the Institute of Physics, Maria Curie-Skłodowska University [11]. The fluence of $\mathrm{In}^{+}$was $3 \times 10^{15}$ ions $/ \mathrm{cm}^{2}$. In this case a current density was not higher than $2.5 \mu \mathrm{A} / \mathrm{cm}^{2}$. The samples were aligned at the angle of $8^{\circ}$ with respect to the ion beam. These experimental conditions provide homogeneous ion implantation and no ion channelling effects. In order to study the oxygen concentration we have used a narrow resonance in the nuclear reaction ${ }^{16} \mathrm{O}\left({ }^{4} \mathrm{He}^{+},{ }^{4} \mathrm{He}^{+}\right){ }^{16} \mathrm{O}$ [12]. The nuclear resonance at $3.045 \mathrm{MeV}$ has a rather large backscattering cross-section (about 16 times greater than for the Rutherford scattering) and the resonance FWHM is only about $10 \mathrm{keV}$. These measurements have been performed at the Van de Graaff accelerator in JINR (Dubna, Russia). During this experiment the energy of the incidence beam of ${ }^{4} \mathrm{He}^{+}$ions was varied in small increments (of about $5 \mathrm{keV}$ ) in the range from $3.040 \mathrm{MeV}$ to $3.056 \mathrm{MeV}$. For this range, the energy spectra of ${ }^{4} \mathrm{He}^{+}$ions scattered under the angle of $170^{\circ}$ with respect to the incident beam were collected. The target was inclined to the beam at the angle of $15^{\circ}$ in all cases.

The optical parameters (refraction index and extinction coefficient) of modified layers for unimplanted and implanted samples were also measured with ellipsometric method. VASE measurements were performed at room temperature with a variable angle spectroscopic ellipsometer of J.A. Woollam [13]. This instrument works in the configuration of a rotating analyzer ellipsometer.

\footnotetext{
* corresponding author; e-mail: rzodki@ite.waw.pl
} 
The ellipsometric angles $\Psi$ and $\Delta$ were used for wavelengths from $250 \mathrm{~nm}$ to $900 \mathrm{~nm}$ with a step $5 \mathrm{~nm}$. Incidence of angles were $65^{\circ}, 70^{\circ}$ and $75^{\circ}$.

The optical constants as a function of light wavelength of the layers enriched in oxygen and modified by ion implantation with indium were determined.

\section{Results and discussion}

Figures 1 and 2 present the spectra of $\alpha$ particles scattered on the GaAs sample implanted with indium ions of $200 \mathrm{keV}$ energy. The both spectra are similar in the energy range from $1.2 \mathrm{MeV}$ to $2.7 \mathrm{MeV}$ but a difference is observed near the energy $1.1 \mathrm{MeV}$. The line near the

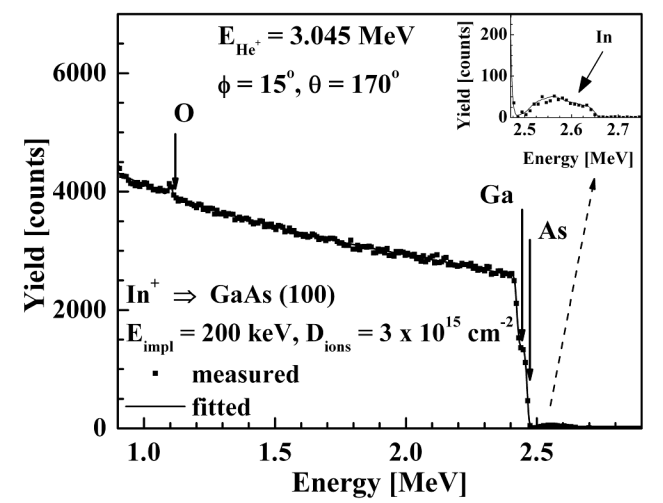

Fig. 1. Random backscattering spectrum for $3.045 \mathrm{MeV} \mathrm{He}{ }^{+}$incident beam on GaAs implanted with indium. In the inset an enlarged part of the spectrum is shown.

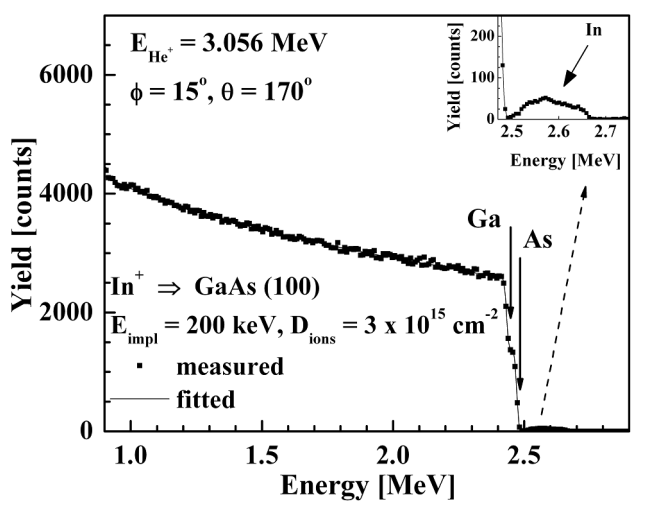

Fig. 2. Backscattering spectrum for $3.056 \mathrm{MeV} \mathrm{He}{ }^{+}$ incident beam on GaAs implanted with indium ions. The inset shows an enlarged part of the spectrum.

energy $1.1 \mathrm{MeV}$ is observed only in Fig. 1, and disappears in Fig. 2. This line is connected with the resonance ${ }^{16} \mathrm{O}\left({ }^{4} \mathrm{He}^{+},{ }^{4} \mathrm{He}^{+}\right){ }^{16} \mathrm{O}$ and it can very precisely qualify, on basis of these data, the thickness of layer enriched with oxygen. This layer is a native-oxides layer which covered the implanted sample in the air. The thickness of

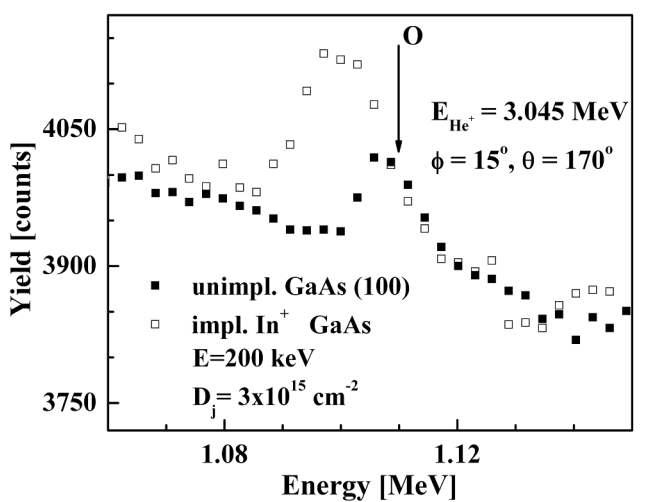

Fig. 3. Backscattering spectra for $3.045 \mathrm{MeV} \mathrm{He}{ }^{+}$incident beam on GaAs implanted with indium and virgin crystal.

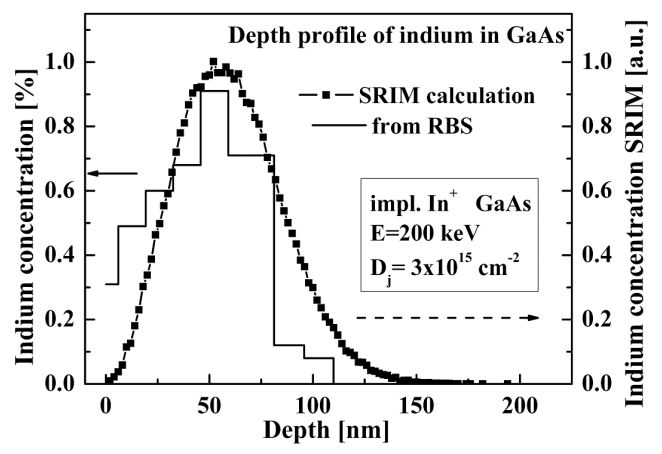

Fig. 4. The depth profiles of indium concentration in implanted GaAs calculated by the help of SRIM 2008 and determined on the basis of RBS measurements.

this layer is $6.0 \mathrm{~nm}$. The same measurements were executed for unimplanted GaAs. The results of this study have been compared with data measured for GaAs implanted with $\mathrm{In}^{+}$and are presented in Fig. 3. It is observed in Fig. 3 that the line in the spectrum collected for virgin GaAs compared with the spectrum measured for implanted sample is narrower and its height is smaller. This effect can be explained by decrease of oxygen concentration in the near surface layer and its thickness. The thickness of the native oxides covering unimplanted GaAs is $2.2 \mathrm{~nm}$. The depth profile of indium concentration obtained on the basis of measurements with RBS and NRA, and SIMRA computer code were used [14]. It is presented in Fig. 4. The atomic concentration of indium is about $1.4 \times 10^{20}$ atoms $/ \mathrm{cm}^{3}$ in the first layer and its thickness is $6 \mathrm{~nm}$. The atoms of oxygen are also present in this layer and their concentration is about $5 \times 10^{21}$ atoms $/ \mathrm{cm}^{3}$. This value is greater than atomic concentration of oxygen in the native oxides on the surface of the virgin GaAs, which it is about $4.0 \times 10^{20}$ atoms $/ \mathrm{cm}^{3}$. These effects can explain that the near surface layer on implanted GaAs has more defects than in unimplanted GaAs and it is more easily oxidized. 

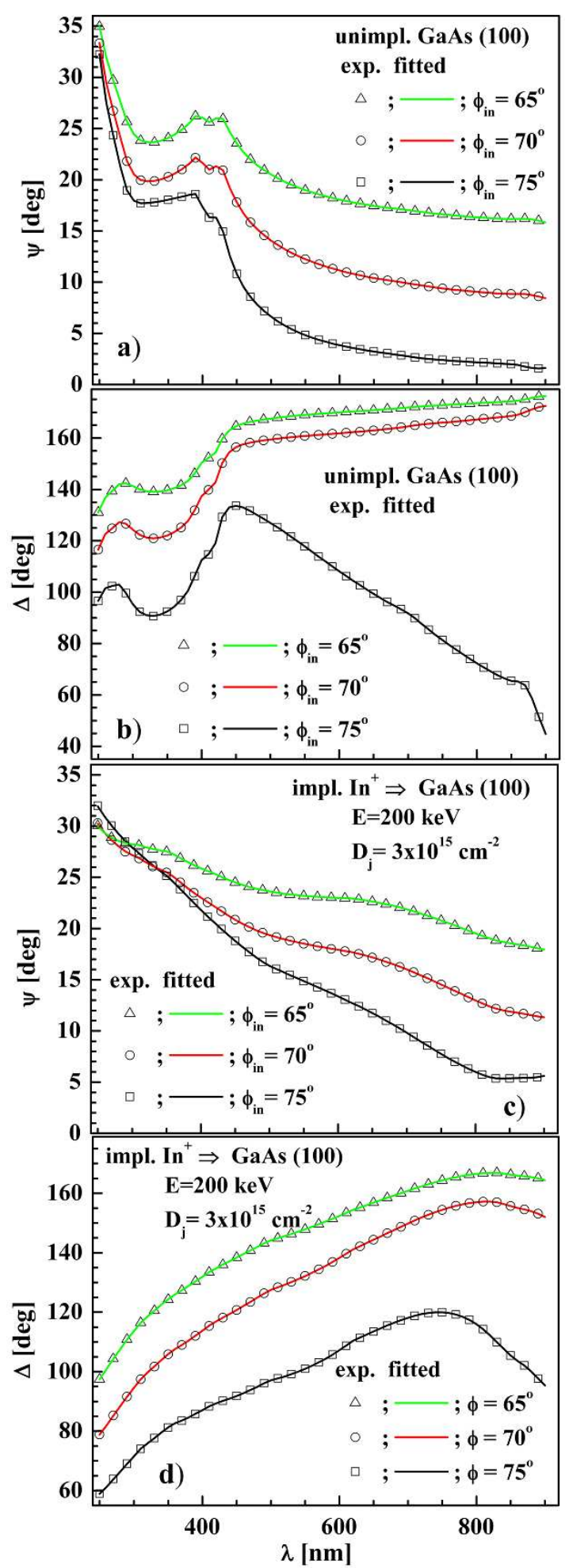

Fig. 5. The spectra of ellipsometric angles $\Psi$ ((a), (c)) and $\Delta((\mathrm{b}),(\mathrm{d}))$ as a function of light wavelength measured at incidence angles $65^{\circ}, 70^{\circ}$ and $75^{\circ}$ for GaAs before and after implantation with indium.
The values of ellipsometric angles as a function of the light wavelength for three incidence angles $65^{\circ}, 70^{\circ}$ and $75^{\circ}$ were measured for crystalline GaAs and GaAs after implantation. The results of measurements and the best fitting of $\Psi$ and $\Delta$ are presented in Fig. 5. The local structures, near $\lambda=400 \mathrm{~nm}$ and $500 \mathrm{~nm}$, of the $\Psi$ and $\Delta$ angles are noticed in Fig. $5 \mathrm{a}$ and Fig. $5 \mathrm{~b}$, respectively. They are presented in the spectra measured for the virgin GaAs and they disappeared in the spectra measured for implanted GaAs (Fig. 5c and d). These structures confirm a good quality of the crystallographic structures of the studied material. The process of ion implantation caused the amorphism of the near surface layer and the optical constants of the ion-irradiated GaAs layer are changed. These changes of optical constants caused, in the region from $400 \mathrm{~nm}$ to $800 \mathrm{~nm}$, the rise of wide bands presented in Fig. 5d. Models of three (ambient, native oxide layer, substrate) and four (ambient, native oxide layer, implanted layer, and substrate) phases are used for description of crystalline and implanted GaAs, respectively, in the ellipsometric study. In the ellipsometric calculations it was assumed that the layers of the native oxides covering the studied samples and the implanted layer were homogeneous and parallel [15]. The thickness of the native oxides and implanted layer were determined on the basis of NRA and RBS measurements, respectively. The refraction and extinction coefficients of native oxides. The optical constants of the implanted layer were described by the effective media approximation (EMA) method [16] and it was alike a homogeneous layer. Their thickness was determined and equal to about $99.8 \mathrm{~nm}$ from RBS measurements. This mixture consists of two phases - amorphous and crystalline GaAs. The percent constitution of the implanted layer was determined and it was $98.6 \%$ and $1.4 \%$ of amorphous and crystalline GaAs, respectively.

The refraction and extinction coefficients determined for GaAs layer implanted with Indium were presented in Fig. 6. In this Figure, the plateau of refractive index at the value of 4.3578 in the range of $600-900 \mathrm{~nm}$ was observed. The effects can be explained as a formation during the implantation of the homogeneous disorder in GaAs. In the last step of this study there were determined the optical constants of the native oxides covering the implanted layer.

These values were compared with data sizes describing oxides covering the crystalline GaAs (see Fig. 7). Two effects were observed in Fig. 7a and b. The first is that the values of refraction indexes increased after implantation in the whole range of studied spectrum ( $\lambda$ from $250 \mathrm{~nm}$ to $920 \mathrm{~nm}$ ). The next one is noticed in Fig. 7b. Two bands are observed in the spectrum determined for oxides covering the surface of implanted samples, near the light wavelengths of $400 \mathrm{~nm}$ and $480 \mathrm{~nm}$. This effect provides that the present oxides are absorbing and probably they are a mixture of native oxides on crystalline GaAs, amorphous GaAs and InAs. 


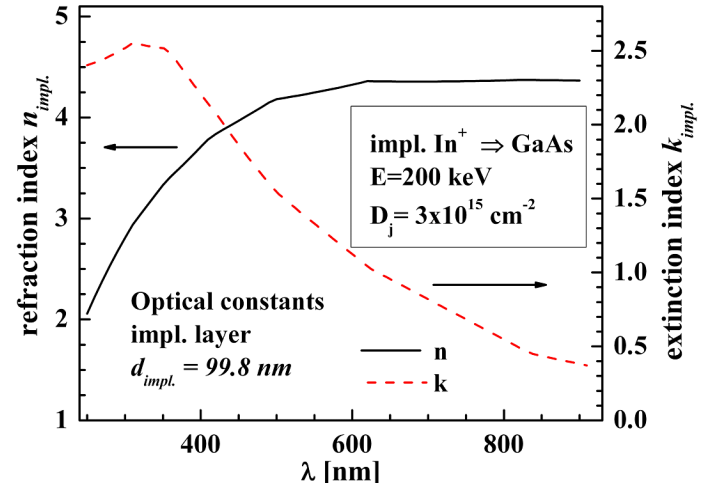

Fig. 6. The spectra of refraction and extinction coefficients determined for GaAs layer implanted with indium.

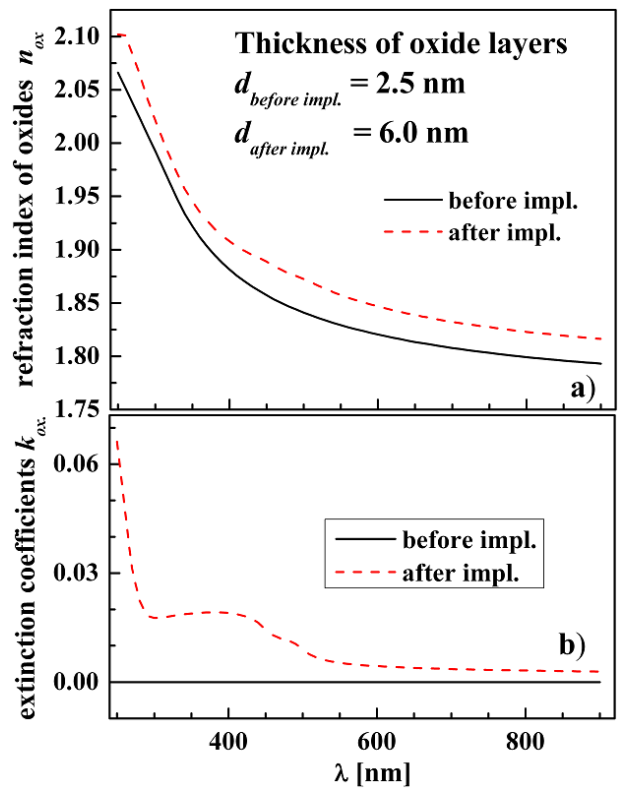

Fig. 7. The spectra of refraction (a) and extinction (b) coefficients determined for native oxides covering the GaAs layer impladnted with indium ions and for oxides covering the crystalline GaAs.

\section{Conclusions}

On the basis of the investigations it can be concluded that the oxidation process of GaAs surface implanted with In ions causes the creation of the thicker layer absorbing natural oxide layer.

\section{References}

[1] B. Breeger, E. Wendler, C. Schubert, W. Wesch, Nucl. Instrum. Methods Phys. Res. B 161, 415 (2000).

[2] M. Kulik, F.F. Komarov, D. Maczka, Vacuum 63, 755 (2001).

[3] M. Webb, C. Jeynes, R. Gwilliam, A. Royle, B. Sealy, Nucl. Instrum. Methods Phys. Res. B 249, 429 (2006).

[4] M. Kulik, F.F. Komarov, D. Maczka, Acta Phys. Pol. A 96, 131 (1999).

[5] M. Kulik, W. Rzodkiewicz, J. Zuk, F.F. Komarov, Przegląd Elektrotechniczny 84, 199 (2008).

[6] H. Lee, R.R. Lowe-Webb, W. Yang, P.C. Sercel, Appl. Phys. Lett. 71, 2325 (1997).

[7] M. Kulik, A.P. Kobzev, D. Jaworska, J. Zuk, J. Filiks, Vaccum 81, 1124 (2007).

[8] M. Fred, T. Lohner, Gy. Viskelethy, E. Jaroli, G. Mezey, J. Gyulai, M. Somorgyi, H. Kerkow, Thin Solid Films 116, 23 (1986).

[9] M. Kulik, K. Jezierski, Acta Phys. Pol. A 75, 413 (1989).

[10] M. Kulik, So. Saied, J. Liśkiewicz, D. Mączka, Nukleonika 44, 167 (1990).

[11] D. Maczka, A. Latuszynski, R. Kuduk, J. Partzka, Nucl. Instrum. Methods Phys. Res. B 21, 521 (1987).

[12] J.R. Cameron, Phys. Rev. 90, 839 (1953).

[13] J.A. Woolam, Guide to Using WASE 32, software for Vase and M44 Ellipsometers.

[14] http://www.rzg.mpg.de/ mam/Version5.html .

[15] M. Kulik, J. Herec, J. Romanek, Vacuum 63, 761 (2001).

[16] H.G. Tompkins, User's Guide to Ellipsometry, Academic Press, Boston 1993. 